\title{
The Role of TPA I/D and PAI-1 4G/5G Polymorphisms in Multiple Sclerosis
}

\author{
Maja Živković, ${ }^{1}$ Nada Starčević Čizmarević, ${ }^{2}$ Luca Lovrečić, ${ }^{3}$ Inge Klupka-Sarić, ${ }^{4}$ \\ Aleksandra Stanković, ${ }^{1}$ Iva Gašparović, ${ }^{5}$ Polona Lavtar, ${ }^{3}$ Evica Dinčić, ${ }^{6}$ Ljiljana Stojković, ${ }^{1}$ \\ Gorazd Rudolf, ${ }^{3}$ Saša Šega Jazbec, ${ }^{7}$ Olivio Perković, ${ }^{5}$ Osman Sinanović, ${ }^{8}$ Juraj Sepčić, ${ }^{9}$ \\ Miljenko Kapović, ${ }^{2}$ Borut Peterlin, ${ }^{3}$ and Smiljana Ristić, ${ }^{2,5}$ \\ ${ }^{1}$ Laboratory for Radiobiology and Molecular Genetics, "Vinča" Institute of Nuclear Sciences, \\ University of Belgrade, 11000 Belgrade, Serbia \\ ${ }^{2}$ Department of Biology and Medical Genetics, School of Medicine, University of Rijeka, 51000 Rijeka, Croatia \\ ${ }^{3}$ Clinical Institute of Medical Genetics, University Medical Centre, 1000 Ljubljana, Slovenia \\ ${ }^{4}$ Department of Neurology, School of Medicine, University of Mostar, 88000 Mostar, Bosnia and Herzegovina \\ ${ }^{5}$ Department of Neurology, Clinical Hospital Center Rijeka, 51000 Rijeka, Croatia \\ ${ }^{6}$ Neurology Clinic, Military Medical Academy, 11000 Belgrade, Serbia \\ ${ }^{7}$ Department of Neurology, University Medical Centre, 1000 Ljubljana, Slovenia \\ ${ }^{8}$ Department of Neurology, School of Medicine, University of Tuzla, 75000 Tuzla, Bosnia and Herzegovina \\ ${ }^{9}$ Postgraduate Studies, School of Medicine, University of Rijeka, 51000 Rijeka, Croatia
}

Correspondence should be addressed to Smiljana Ristić; smiljana.ristic@medri.uniri.hr

Received 4 February 2014; Accepted 31 March 2014; Published 16 April 2014

Academic Editor: Ralf Lichtinghagen

Copyright (C) 2014 Maja Živković et al. This is an open access article distributed under the Creative Commons Attribution License, which permits unrestricted use, distribution, and reproduction in any medium, provided the original work is properly cited.

\begin{abstract}
Background. Previous studies have shown impaired fibrinolysis in multiple sclerosis (MS) and implicated extracellular proteolytic enzymes as important factors in demyelinating neuroinflammatory disorders. Tissue-type plasminogen activator ( $\mathrm{t}$-PA) and its inhibitor (PAI-1) are key molecules in both fibrinolysis and extracellular proteolysis. In the present study, an association of the TPA Alu I/D and PAI-1 4G/5G polymorphisms with MS was analyzed within the Genomic Network for Multiple Sclerosis (GENoMS). Methods. The GENoMS includes four populations (Croatian, Slovenian, Serbian, and Bosnian and Herzegovinian) sharing the same geographic location and a similar ethnic background. A total of 885 patients and 656 ethnically matched healthy blood donors with no history of MS in their families were genotyped using PCR-RFLP. Results. TPA DD homozygosity was protective $(\mathrm{OR}=0.79,95 \%$ CI $0.63-0.99, P=0.037)$ and PAI 5G5G was a risk factor for MS (OR $=1.30,95 \% \mathrm{CI} 1.01-1.66, P=0.038)$. A significant effect of the genotype/carrier combination was detected in $5 \mathrm{G} 5 \mathrm{G} / \mathrm{I}$ carriers $(\mathrm{OR}=1.3995 \% \mathrm{CI} 1.06-1.82, P=0.017)$. Conclusions. We found a significantly harmful effect of the combination of the PAI-1 5G/5G genotype and TPA I allele on MS susceptibility, which indicates the importance of gene-gene interactions in complex diseases such as MS.
\end{abstract}

\section{Introduction}

Multiple sclerosis (MS) is a complex inflammatory demyelinating disease of the central nervous system (CNS) with both genetic and environmental contributing factors. The underlying cause of the disease is thought to be the cumulative effect of multiple genetic variants throughout the genome, each making a modest contribution to the onset and progression of the disease. However, despite great progress in detecting risk loci for MS using high-throughput technologies over the last decade [1], much remains to be discovered about the genetics of MS [2].

In recent years, increasing evidence has pointed to the potential role of fibrinolysis in the pathogenesis of MS. Specifically, the characteristic inflammation, focal demyelination, and axonal degeneration in MS occur after disruption 
TABLE 1: Characteristics of MS patients.

\begin{tabular}{lcccc}
\hline Parameter & Serbian $(n=382)$ & BH $(n=170)$ & Croatian/Slovenian $(n=333)$ & Pooled samples $(n=885)$ \\
\hline Gender (female/male) & $241 / 141(63.1 / 36.9)$ & $120 / 50(70.6 / 29.4)$ & $239 / 94(71.7 / 28.3)$ & $600 / 285(67.8 / 32.2)$ \\
Age of disease onset (years) & $29.2 \pm 7.0$ & $30.8 \pm 8.0$ & $29.4 \pm 8.9$ & $30.1 \pm 8.0$ \\
MSSS & $5.4 \pm 2.5$ & $4.8 \pm 2.8$ & $4.9 \pm 2.7$ & $5.2 \pm 2.6$ \\
\hline
\end{tabular}

MSSS: Multiple Sclerosis Severity Score.

Values are expressed as mean \pm SD.

of the blood-brain barrier (BBB) and entry of serum proteins, including fibrinogen, into the CNS [3]. Extracellular proteolysis represents a potent and irreversible mechanism modulating the extracellular matrix and tissue remodeling, which can affect the breakdown of the BBB. Extracellular proteolytic enzymes have been implicated as important factors in demyelinating neuroinflammatory disorders such as MS [4]. The enzymes of the plasminogen activators/plasmin (PA) system are involved in both fibrinolysis and extracellular proteolyses.

The key molecules in the PA system are tissue-type plasminogen activator (t-PA) and its inhibitor (PAI-1). Due to the formation of t-PA and inhibitor (e.g., PAI) complexes, the fibrinolytic potential in demyelinating MS lesions is greatly diminished [5]. The limited availability of t-PA because of the formation of the t-PA/PAI-1 complexes is assumed to reduce the ability of $\mathrm{t}-\mathrm{PA}$ receptors to produce plasmin, which further diminishes the fibrinolytic capacity in MS lesions, possibly resulting in increased axonal fibrin deposition and neurodegeneration $[6,7]$. On the other hand, it may represent a mechanism to remove fibrin deposits, which are then cleared through internalization by macrophages.

In experimental autoimmune encephalomyelitis (EAE), deletion of the TPA gene results in an earlier appearance and more severe form of disease associated with high levels of PAI-1 and inefficient fibrin removal [7]. Recent studies have shown that the PAI-1 ${ }^{-}$knockout $\left(\mathrm{PAI}-1^{-/-}\right)$mice have reduced disease incidence, a milder clinical picture, lack of clinical relapse, and reduced neuroinflammation, as well as less axonal damage than wild-type (wt) mice [8].

An Alu-repeat insertion/deletion (I/D) in intron 8 of the TPA gene and the $4 \mathrm{G} / 5 \mathrm{G}$ polymorphism in the $5^{\prime}$ untranslated region at -675 of the PAI-1 gene are known, as are differences in the productivity of these proteins between genotypes. The t-PA release rates were markedly higher in subjects homozygous for the I allele [9], whereas individual homozygous for the $4 \mathrm{G}$ allele has a $25 \%$ higher plasma PAI-1 concentration than those with the 5G5G genotype [10].

To date, only two studies have investigated the correlation between these polymorphisms and MS [11, 12].

Because of technical limitations, the insertion/deletion polymorphism could not be analyzed in recent GWAS studies.

The aim of the present study was to investigate the role of the TPA I/D and PAI-1 4G/5G polymorphisms in a new MS study population and perform a meta-analysis of previous studies $[11,12]$. This study was conducted within the Genomic Network for Multiple Sclerosis (GENoMS), which includes four populations (Croatian, Slovenian, Serbian, and Bosnian and Herzegovinian $[\mathrm{BH}]$ ) that share the same geographic location and a similar ethnic background, a Slavic origin.

\section{Materials and Methods}

2.1. Subjects. Cohorts of MS patients collected in Serbia and $\mathrm{BH}$ were included in the study. All patients fulfilled the criteria for clinically definite MS [13] and the course of the disease was classified based on clinical data [14]. Patients with relapsing-remitting (RR) or a secondary progressive (SP) course were grouped as bout-onset and analyzed separately from patients with the primary progressive (PP) form. Disease severity was estimated using the Multiple Sclerosis Severity Score (MSSS) [15], which corrects the Expanded Disability Status Scale (EDSS) [16] for disease duration.

A total of 382 Serbian patients (141 males and 241 females) were recruited from the Department of Neurology of the Military Medical Academy (MMA), including 372 (97.4\%) patients with bout-onset MS and 10 (2.6\%) with PPMS. The mean age at onset was $29.2 \pm 7.0$ years and the mean MSSS was $5.38 \pm 2.52$. The Serbian controls were 318 healthy volunteers without a history of MS in their families and of the same ethnic origin as the MS patients.

The $\mathrm{BH}$ dataset consisted of 170 patients (50 males and 120 females) recruited consecutively from the Department of Neurology, School of Medicine, University of Mostar and Department of Neurology, School of Medicine, University of Tuzla, and 170 healthy volunteers of matched ethnicity (Bosnians, Croatians, Serbians). Among the patients, 160 (94.1\%) had bout-onset MS and 10 (5.9\%) had PPMS. The average age at onset was $30.8 \pm 8.0$ years and the mean MSSS was $4.79 \pm 2.81$.

For pooled analyses, we included the Croatian and Slovenian subjects, which were the study groups used in a recently published study [12]. The $333 \mathrm{MS}$ patients (94 males, 239 females), including 308 (92.5\%) with bout-onset MS and 25 (7.5\%) with PPMS, had a mean age at onset of $29.4 \pm 8.9$ years and mean MSSS of $4.94 \pm 2.73$. A total of 368 ethnically matched healthy blood donors with no history of MS in their families represented the control group.

Table 1 provides a description of the MS groups included in the study.

2.2. Genotyping. Genomic DNA was extracted from whole blood using standard procedures. Genotyping was performed in the Molecular Genetics Laboratory (Department of Biology and Medical Genetics, School of Medicine, Rijeka) using PCR (for the TPA Alu I/D polymorphism) or PCR-RFLP 
(for the PAI-1 4G/5G polymorphism) as described previously $[9,17]$.

2.3. Statistical Analysis. Statistical analyses were carried out using Statistical Software package for Windows 7.1 (StatSoft, Inc.). Differences in the allele and genotype frequency distribution between the studied groups, as well as deviation from Hardy-Weinberg equilibrium, were estimated by the chi-square $\left(\chi^{2}\right)$ test. A logistic regression analysis expressed in terms of an adjusted odds ratio (OR), and 95\% confidence interval (CI) was used as a measure of the strength of the association between the TPA I/D and PAI-1 4G/5G polymorphisms and susceptibility to MS.

To assess the relationship between studied polymorphisms and MS, we performed a meta-analysis of the four datasets, comprising Serbian, BH, Croatian, Slovenian [12], and Finnish subjects [11]. Subjects were classified by diagnostic category (patients and controls) and number of events (the presence of the PAI-1 5G5G genotype or the presence of the PAI-1 5G5G/TPA I carrier combination). The strength of the association was summarized using the OR in which PAI-1 5G5G homozygosity or PAI-1 5G5G/TPA I carriership was assigned as a risk factor for MS. Each of the cohorts was analyzed separately, and the significance of the pooled ORs was determined using the $z$-test. The heterogeneity of the group of ORs was assessed using the $\chi^{2}$-based $Q$-statistic test. Meta-analyses were conducted using Comprehensive MetaAnalysis software version 2.0 (Biostat International, Inc.).

Analysis of variance (ANOVA) and appropriate post hoc tests were used to test the relationships between the polymorphisms and continuous variables. In all of the tests, differences with two-tailed $P \leq 0.05$ were considered significant. Multivariate regression analysis was performed to assess the influence of genetic and clinical parameters on disease onset, clinical course, and MS progression.

2.4. Ethics Statement. The study was approved by the respective local ethics committees in all countries participating in the study: Ethics Committee of the MMA, Serbia (reference number: 29/2-05), Ethics committee of the Clinical Hospital Mostar (reference number: 3590/07), the Ethics committee of the University Clinical Hospital Tuzla, Bosnia, and Herzegovina (reference number: 01/3-37-4-7683/07), Ethics committee at School of Medicine at University of Rijeka, Croatia (reference number: 641-01/06-01/66), and the Slovenian National medical ethics committee (reference number: 90/08/12). All participants signed a written informed consent. For those patients who have had a compromised capacity to consent (Folstein Mini-Mental State Examination (MMSE) score below 20) informed consent was signed by the nearest relative or guardian.

\section{Results}

3.1. TPA I/D and PAI-1 4G/5G Genotypes and Alleles in MS Patients and Controls. The Serbian and $\mathrm{BH}$ case-control groups were analyzed separately and also pooled with Croatian and Slovenian samples [12] following the demonstration of a lack of significant heterogeneity. The distributions of allele and genotype frequencies are provided in Table 2. The genotype distributions among MS patients and controls were compatible with Hardy-Weinberg expectations for both the Serbian and BH cohorts. In the Serbian cohort, PAI-1 5G5G homozygosity $(\mathrm{OR}=1.54,95 \%$ CI $1.04-2.28, P=0.032)$ was positively associated with MS, and 4G5G heterozygosity $(\mathrm{OR}=0.68,95 \%$ CI $0.50-0.92, P=0.012)$ was negatively associated with MS compared to controls. The distribution of the TPA I/D polymorphism was not significantly different in patients compared to controls in the Serbian group. No significant differences were found in the genotype and allele frequency distribution for the polymorphisms in $\mathrm{BH}$ patients and controls.

3.2. TPA I/D and PAI-1 4G/5G Genotypes in Pooled MS Samples and Meta-Analysis. Comparisons of allele and genotype frequencies between pooled MS patients and controls (Serbian, BH, Croatian, and Slovenian, Table 2) did not reveal significant associations, but we found a trend towards a decreased risk of MS in patients with the TPA DD genotype $(\mathrm{OR}=0.80,95 \%$ CI $0.65-1.00, P=0.050)$ and an increased risk of $\mathrm{MS}$ in patients with the PAI-1 5G5G genotype $(\mathrm{OR}=$ 1.27, 95\% CI 0.97-1.61, $P=0.054)$.

Although the crude association of the TPA and PAI-1 polymorphisms with MS in a pooled sample (Serbian, BH, Croatian, and Slovenian) did not reveal a significant association after adjusting the ORs for gender, the TPA DD genotype was shown to be significantly protective $(\mathrm{OR}=0.79,95 \% \mathrm{CI}$ $0.63-0.99, P=0.037$ ) and the PAI-1 5G5G genotype was shown to be a significant risk factor $(\mathrm{OR}=1.30,95 \% \mathrm{CI}$ 1.01-1.66, $P=0.038$ ) for MS (Figure 1). The meta-analysis, which also included the results from a Finnish study [11], confirmed the significant risk for MS among PAI-1 5G5G homozygotes $(\mathrm{OR}=1.33,95 \% \mathrm{CI} 1.06-1.67, z=2.450, P=$ $0.014, P_{\text {heterogeneity }}=0.143$; Figure 2 ) compared to the carriers of the $4 \mathrm{G}$ allele.

3.3. Combination of TPA I/D and PAI-1 4G/5G Genotypes in Pooled MS Samples and Meta-Analysis. We analyzed the genotype combinations and/or allele carriership for the TPA I/D and PAI-1 4G/5G polymorphisms in a pooled study sample. The frequency analysis of these combinations is shown in Table 3. The only significant effect of the genotype/allele carrier combination was detected in 5G5G/I carriers (both of these polymorphisms separately carry a significant risk for MS), with an even higher and more significant OR for MS than in the separate analysis $(\mathrm{OR}=1.39$ 95\% CI 1.06-1.82, $P=0.017)$. The meta-analysis confirmed this result $(\mathrm{OR}=$ $1.38,95 \%$ CI 1.05-1.82, $z=2.328, P=0.020, P_{\text {heterogeneity }}=$ 0.102; Figure 3). We did not find a significant difference in the distribution of the genotypes and alleles of the tested genes after stratifying by gender.

3.4. TPA I/D and PAI-1 4G/5G Genotypes and Clinical Characteristics of MS. We did not find a significant association between the separate or combined effects of the TPA I/D or PAI-1 4G/5G genotypes and disease course (bout onset 


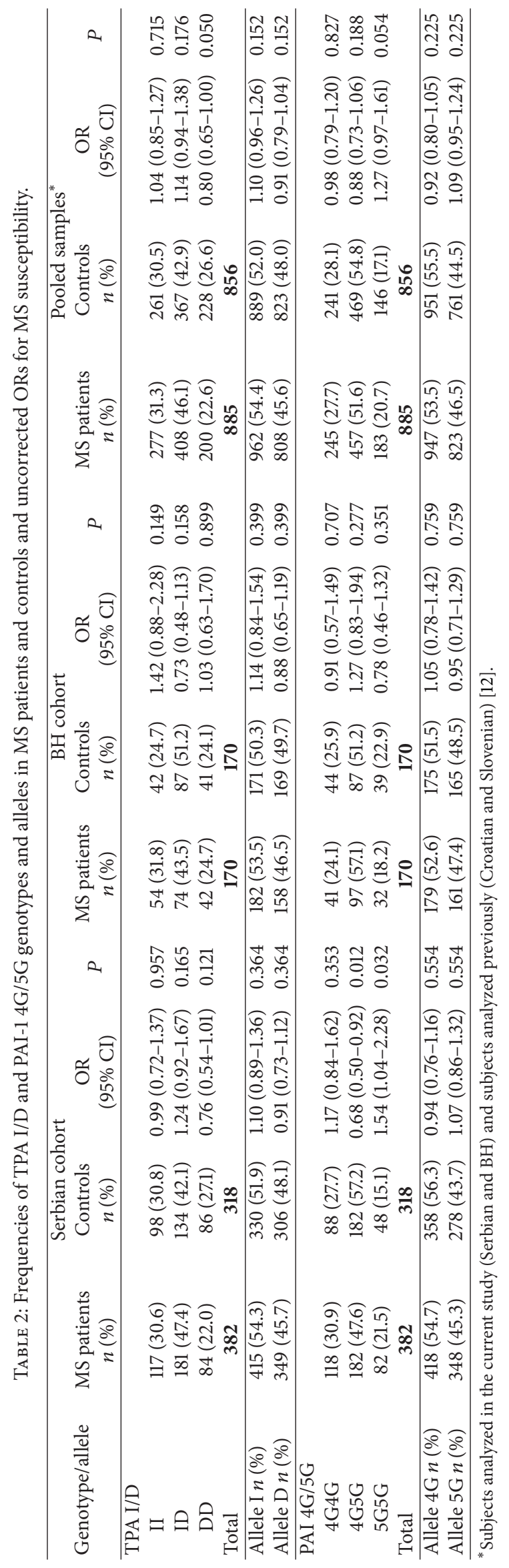




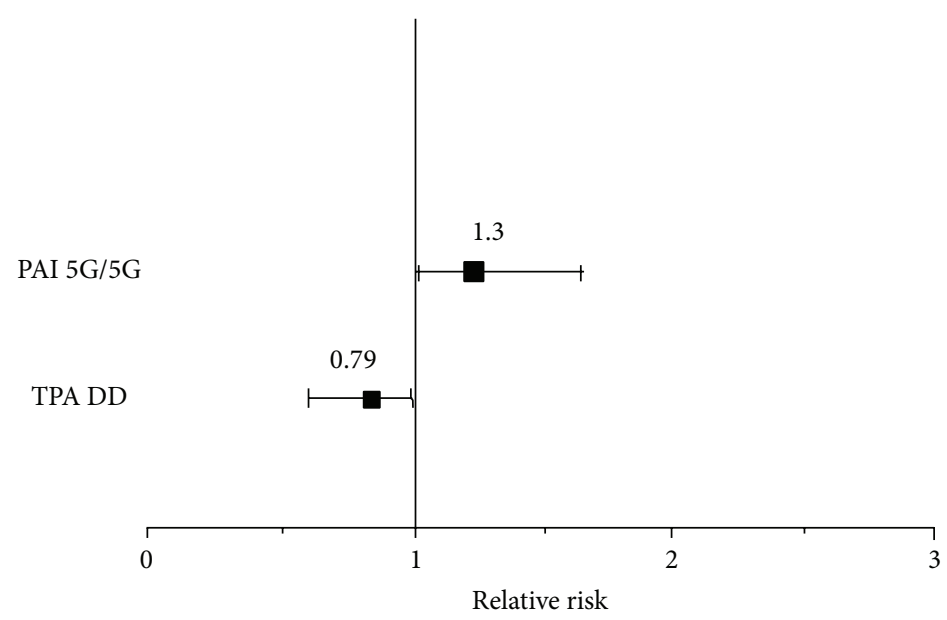

Figure 1: The separate effects of the PAI-1 5G/5G and TPA D/D genotypes on MS susceptibility in a pooled sample of 885 MS patients. Data are presented as the OR adjusted for gender. The error bars represent the 95\% CI. TPA D/D, OR $=0.80,95 \%$ CI $0.65-1.00, P=0.050$; PAI-1 5G/5G, OR=1.27, 95\% CI 0.97-1.61, $P=0.054$.

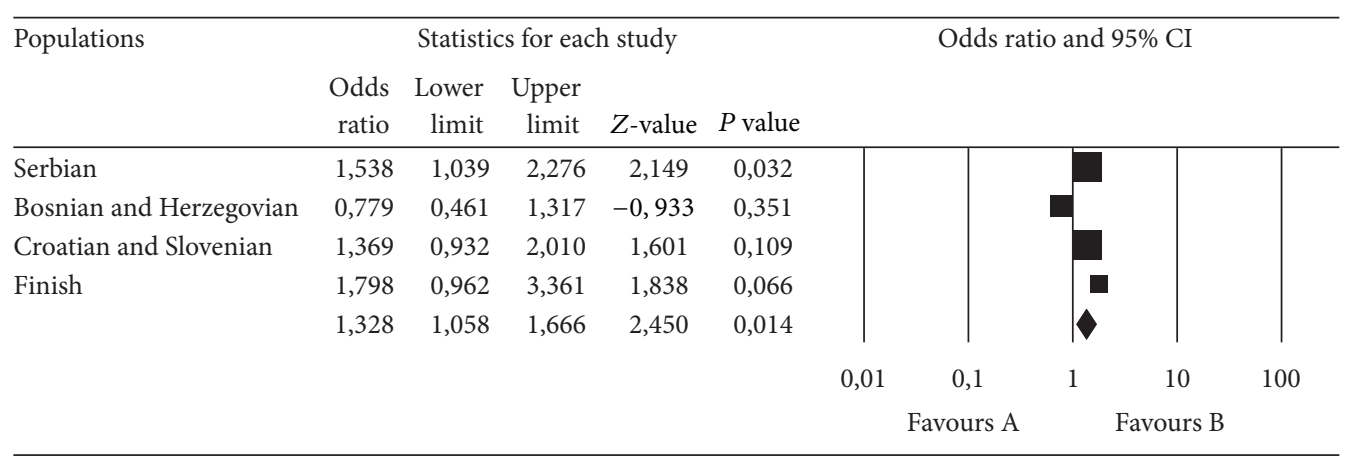

FIGURE 2: Meta-analysis of the four case-control studies of MS and the PAI-1 4G/5G polymorphism. Comparisons were made between the $5 \mathrm{G} 5 \mathrm{G}$ and $4 \mathrm{G} 4 \mathrm{G}+4 \mathrm{G} 5 \mathrm{G}$ genotypes. Summary ORs and respective $95 \%$ CIs were calculated using the fixed-effect model.

versus PP), age at disease onset, or MSSS in the Serbian and $\mathrm{BH}$ patient samples separately, or in a pooled sample of MS patients (data not shown).

\section{Discussion}

In this study, we analyzed the influence of the TPA I/D and PAI-1 4G/5G polymorphisms on MS. We found a significant, but opposite, influence of the rare TPA and PAI genotypes on MS susceptibility. The TPA D/D genotype had a protective effect, whereas PAI 5G/5G was a risk factor for MS. The meta-analysis for the PAI-1 polymorphism, which included the previously published results [11], confirmed that 5G/5G represents a significant risk factor for MS.

The investigated polymorphisms have been investigated predominantly in vascular disorders [18], rather than diseases such as MS. The single alleles (e.g., 4G) have been found to have opposite effects in cardiac disease (increased risk) compared to brain ischemia (protective effect).

The $4 \mathrm{G}$ allele has been linked to higher serum levels of PAI-1 [10]. Recent data suggest a functional importance of the PAI-1 4G/5G polymorphism, which could have a neuroprotective effect through increased astrocytic expression of PAI-1 [19]. Haplotypes containing the PAI-1 4G allele have been associated with higher transcriptional activity in astrocytes compared to haplotypes containing 5G [19]. In this study, the 5G5G genotype exhibited a 1.3-times higher risk for MS susceptibility compared to $4 \mathrm{G}$ carriers. In a previous study of Finnish women with MS [11], the 5G5G genotype, which is linked to lower plasma levels of PAI-1 [10], was associated with an increased risk of MS. These results show a consistency in the neuroprotective role of sufficient PAI-1 in the brain through allele-specific regulation of its presence. In addition, the PAI-1 gene is located in the MS susceptibility locus on chromosome 7q21-22 [20]. Only two studies previously looked at the association with MS [11, 12], and GWAS lack data on insertion/deletion polymorphisms, such as the two investigated here.

In the brain, PAI-1 is produced predominantly by astrocytes [21], whereas its main function is to inhibit t-PA [22]. After injury, an excessive amount of t-PA is released into the extracellular space of the brain, which can trigger both neuronal degeneration [23] and disruption ofthe BBB [24]. 
TABLE 3: The uncorrected odds ratios (ORs) for combination genotypes of the TPA I/D and PAI 4G/5G polymorphisms in MS patients and controls.

\begin{tabular}{lccc}
\hline Genotype combinations & MS patients $(n=885)$ & Controls $(n=856)$ & OR (95\% CI) \\
\hline PAI 4G4G/TPA DD & $58(6.6 \%)$ & $71(8.3 \%)$ & $0.78(0.54-1.1)$ \\
PAI 5G5G/TPA DD & $39(4.4 \%)$ & $38(4.4 \%)$ & $1(0.63-1.57)$ \\
PAI 4G carrier/TPA DD & $161(18.2 \%)$ & $185(21.6 \%)$ & $0.167(0.64-1.02)$ \\
PAI 5G5G/TPA I carrier & $144(16.3 \%)$ & $105(12.3 \%)$ & 0.974 \\
\hline
\end{tabular}

\begin{tabular}{|c|c|c|c|c|c|c|c|c|c|c|}
\hline \multirow[t]{2}{*}{ Populations } & \multicolumn{5}{|c|}{ Statistics for each study } & \multicolumn{5}{|c|}{ Odds ratio and 95\% CI } \\
\hline & $\begin{array}{l}\text { Odds } \\
\text { ratio }\end{array}$ & $\begin{array}{c}\text { Lower } \\
\text { limit }\end{array}$ & $\begin{array}{l}\text { Upper } \\
\text { limit }\end{array}$ & $Z$-value & $P$ value & & & & & \\
\hline Croatian and Slovenian & 1,510 & 0,974 & 2,339 & 1,844 & 0,065 & \multirow{6}{*}{0,01} & & & & \\
\hline Serbian & 1,745 & 1,119 & 2,719 & 2,458 & 0,014 & & & & & \\
\hline \multirow[t]{4}{*}{ Bosnian and Herzegovian } & 0,805 & 0,451 & 1,436 & $-0,736$ & 0,462 & & & & & \\
\hline & 1,385 & 1,053 & 1,823 & 2,328 & 0,020 & & & & & \\
\hline & & & & & & & 0,1 & 1 & 10 & 100 \\
\hline & & & & & & & Favours & & our & \\
\hline
\end{tabular}

FIGURE 3: Meta-analysis of the three case-control studies of MS and the PAI-1 4G/5G and TPA I/D polymorphisms. Comparisons were made between the PAI-1 5G5G/TPA I carriers and other genotype/carrier combinations. Summary ORs and respective 95\% CIs were calculated using the fixed-effect model.

Thus, PAI-1 is needed to reduce the deleterious effects of excessive t-PA activity. An increase in brain damage has been shown in PAI-1-deficient mice [25].

In the mouse model of MS, EAE, t-PA has been suggested to have a dual effect. TPA knockout mice exhibit delayed onset of EAE, but the symptoms were more severe and recovery was reduced [26]. This finding suggests that t-PA could have deleterious effects on the initiation of EAE. We found a borderline significance for the protective effect of the TPA D/D genotype in MS patients. The net release rate of $t-$ PA in vivo is significantly higher in the presence of the TPA I/I genotype compared to carriers of the D allele [9].

The increase in plasma t-PA is related to the appearance of EAE symptoms in rats [27]. In the brain, t-PA is localized in the endothelium within regions of neuroinflammation. Monocyte diapedesis through the $\mathrm{BBB}$ depends on t-PA activity, which can induce the breakdown of the tightjunction protein occludin, leading to an increase in the permeability of the interface between the blood and the brain, the neurovascular unit (NVU), and subsequent monocyte infiltration into the CNS [28].

Fibrinolysis is generally regulated by the balance between plasminogen activators and inhibitors. Although t-PA and PAI-1 are not the only players in this process, they are among the most important, and the combination genotype is associated with higher PAI-1 and lower t-PA levels, which could be protective against the deleterious effect of t-PA on $\mathrm{BBB}$ disruption and lymphocyte infiltration in the brain. The 4G/4G D/D combination, which was not common among MS patients $(6.6 \%)$ or controls $(8.3 \%)$, had the protective OR, but was not significant. We found a significantly harmful effect of the combination of the PAI-1 5G/5G genotype and TPA I allele on MS susceptibility. A reasonable explanation could be that the potentially higher t-PA levels and lower PAI-1 levels in carriers of the 5G/5G and I alleles favor the proteolytic function of $\mathrm{t}-\mathrm{PA}$. We did not find any association of the analyzed polymorphisms with clinical parameters or the course of the disease. This finding suggests that the proteolytic function of $\mathrm{t}-\mathrm{PA}$ and its regulation by its major inhibitor is more important for MS onset than its role in fibrinolysis. Because t-PA is biochemically linked to the matrix metalloproteinase (MMP) axis of extracellular proteolysis, it could be important for future studies to examine the relationship between t-PA and MMPs, at the level of the genegene interaction or on the level of the functional interaction, which might include the inflammatory cells that infiltrate the brain through the disrupted BBB.

Although the role of fibrinolysis and extracellular proteolysis has been described in both EAE and MS, this study is among the first to analyze the insertion/deletion polymorphisms in PAI-1 and TPA genes in the context of MS. Validation of our results in other populations and larger sample sizes is needed.

\section{Conclusion}

In conclusion, we found a significantly harmful effect of the PAI-5G/5G genotype on MS in both a study cohort and meta-analysis. A more significant effect on MS susceptibility compared to separate gene analysis was shown for the combination of the PAI-1 5G/5G genotype and TPA I allele. As shown for the other gene loci in MS, these results indicate the importance of gene-gene interactions in complex diseases such as MS. 


\section{Conflict of Interests}

The authors declare that there is no conflict of interests regarding the publication of this paper.

\section{Acknowledgments}

The study was funded by the Serbian Ministry of Education and Science Grant no. 175085 (http://www.mpn.gov.rs), by a Grant from the University of Rijeka, Republic of Croatia, 13.06.1.1.10 (http://www.uniri.hr), and by a Grant no. J33628 from the National Research Agency of the Republic of Slovenia (http://www.arrs.gov.si). The authors express special gratitude to the participants in this study.

\section{References}

[1] S. Sawcer, G. Hellenthal, M. Pirinen et al., "Genetic risk and a primary role for cell-mediated immune mechanisms in multiple sclerosis," Nature, vol. 476, no. 7359, pp. 214-219, 2011.

[2] S. E. Baranzini and D. Nickles, "Genetics of multiple sclerosis: swimming in an ocean of data," Current Opinion in Neurology, vol. 25, no. 3, pp. 239-245, 2012.

[3] E. East, D. Baker, G. Pryce, H. R. Lijnen, M. L. Cuzner, and D. Gverić, "A role for the plasminogen activator system in inflammation and neurodegeneration in the central nervous system during experimental allergic encephalomyelitis," The American Journal of Pathology, vol. 167, no. 2, pp. 545-554, 2005.

[4] T. Teesalu, A. E. Hinkkanen, and A. Vaheri, "Coordinated induction of extracellular proteolysis systems during experimental autoimmune encephalomyelitis in mice," The American Journal of Pathology, vol. 159, no. 6, pp. 2227-2237, 2001.

[5] D. Gveric, R. Hanemaaijer, J. Newcombe, N. A. Van Lent, C. F. M. Sier, and M. L. Cuzner, "Plasminogen activators in multiple sclerosis lesions implications for the inflammatory response and axonal damage," Brain, vol. 124, no. 10, pp. 1978-1988, 2001.

[6] D. Gverić, B. Herrera, A. Petzold, D. A. Lawrence, and M. L. Cuzner, "Impaired fibrinolysis in multiple sclerosis: a role for tissue plasminogen activator inhibitors," Brain, vol. 126, no. 7, pp. 1590-1598, 2003.

[7] D. Gverić, B. M. Herrera, and M. L. Cuzner, "tPA receptors and the fibrinolytic response in multiple sclerosis lesions," The American Journal of Pathology, vol. 166, no. 4, pp. 1143-1151, 2005.

[8] E. East, D. Gverić, D. Baker, G. Pryce, H. R. Lijnen, and M. L. Cuzner, "Chronic relapsing experimental allergic encephalomyelitis (CREAE) in plasminogen activator inhibitor-1 knockout mice: the effect of fibrinolysis during neuroinflammation," Neuropathology and Applied Neurobiology, vol. 34, no. 2, pp. 216-230, 2008.

[9] C. Jern, P. Ladenvall, U. Wall, and S. Jern, "Gene polymorphism of t-PA is associated with forearm vascular release rate of t-PA," Arteriosclerosis, Thrombosis, and Vascular Biology, vol. 19, no. 2, pp. 454-459, 1999.

[10] P. Eriksson, B. Kallin, F. M. Van't Hooft, P. Bavenholm, and A. Hamsten, "Allele-specific increase in basal transcription of the plasminogen-activator inhibitor 1 gene is associated with myocardial infarction," Proceedings of the National Academy of Sciences of the United States of America, vol. 92, no. 6, pp. 18511855, 1995.
[11] M. Luomala, I. Elovaara, M. Ukkonen, T. Koivula, and T. Lehtimäki, "Plasminogen activator inhibitor 1 gene and risk of MS in women," Neurology, vol. 54, no. 9, pp. 1862-1864, 2000.

[12] L. Lovrečić, S. Ristić, N. Starčević-Čizmarević et al., "PAI and TPA gene polymorphisms in multiple sclerosis," Multiple Sclerosis Journal, vol. 14, no. 2, pp. 243-247, 2008.

[13] C. H. Polman, S. C. Reingold, B. Banwell et al., "Diagnostic criteria for multiple sclerosis: 2010 revisions to the McDonald criteria," Annals of Neurology, vol. 69, no. 2, pp. 292-302, 2011.

[14] F. D. Lublin and S. C. Reingold, "Defining the clinical course of multiple sclerosis: results of an international survey," Neurology, vol. 46, no. 4, pp. 907-911, 1996.

[15] R. H. Roxburgh, S. R. Seaman, T. Masterman et al., "Multiple sclerosis severity score: using disability and disease duration to rate disease severity," Neurology, vol. 64, no. 7, pp. 1144-1151, 2005.

[16] J. F. Kurtzke, "Rating neurologic impairment in multiple sclerosis: an expanded disability status scale (EDSS)," Neurology, vol. 33, no. 11, pp. 1444-1452, 1983.

[17] M. Margaglione, E. Grandone, G. Cappucci et al., "An alternative method for PAI-1 promoter polymorphism (4G/5G) typing," Thrombosis and haemostasis, vol. 77, no. 3, pp. 605-606, 1997.

[18] Z. Ye, E. H. C. Liu, J. P. T. Higgins et al., "Seven haemostatic gene polymorphisms in coronary disease: meta-analysis of 66 155 cases and 91307 controls," The Lancet, vol. 367, no. 9511, pp. 651-658, 2006.

[19] K. Hultman, A. Tjärnlund-Wolf, J. Odeberg, P. Eriksson, and C. Jern, "Allele-specific transcription of the PAI-1 gene in human astrocytes," Thrombosis and Haemostasis, vol. 104, no. 5, pp. 998-1008, 2010.

[20] J. L. Haines, M. Ter-Minassian, A. Bazyk et al., "A complete genomic screen for multiple sclerosis underscores a role for the major histocompatability complex. The Multiple Sclerosis Genetics Group," Nature Genetics, vol. 13, no. 4, pp. 469-471, 1996.

[21] I. Gravanis and S. E. Tsirka, "Tissue plasminogen activator and glial function," Glia, vol. 49, no. 2, pp. 177-183, 2005.

[22] J. Chmielewska, M. Ranby, and B. Wiman, "Kinetics of the inhibition of plasminogen activators by the plasminogen-activator inhibitor: evidence for 'second-site' interactions," Biochemical Journal, vol. 251, no. 2, pp. 327-332, 1988.

[23] S. E. Tsirka, A. D. Rogove, T. H. Bugge, J. L. Degen, and S. Strickland, "An extracellular proteolytic cascade promotes neuronal degeneration in the mouse hippocampus," Journal of Neuroscience, vol. 17, no. 2, pp. 543-552, 1997.

[24] M. Yepes, M. Sandkvist, E. G. Moore, T. H. Bugge, D. K. Strickland, and D. A. Lawrence, "Tissue-type plasminogen activator induces opening of the blood-brain barrier via the LDL receptor-related protein," Journal of Clinical Investigation, vol. 112, no. 10, pp. 1533-1540, 2003.

[25] N. Nagai, I. Vanlinthout, and D. Collen, "Comparative effects of tissue plasminogen activator, streptokinase, and staphylokinase on cerebral ischemic infarction and pulmonary clot lysis in hamster models," Circulation, vol. 100, no. 25, pp. 2541-2546, 1999.

[26] W. Lu, M. Bhasin, and S. E. Tsirka, "Involvement of tissue plasminogen activator in onset and effector phases of experimental allergic encephalomyelitis," Journal of Neuroscience, vol. 22, no. 24, pp. 10781-10789, 2002. 
[27] A. Reijerkerk, G. Kooij, S. M. A. van der pol et al., "Tissue-type plasminogen activator is a regulator of monocyte diapedesis through the brain endothelial barrier," Journal of Immunology, vol. 181, no. 5, pp. 3567-3574, 2008.

[28] S. Lemarchant, F. Docagne, E. Emery, D. Vivien, C. Ali, and M. Rubio, "TPA in the injured central nervous system: different scenarios starring the same actor?" Neuropharmacology, vol. 62, no. 2, pp. 749-756, 2012. 


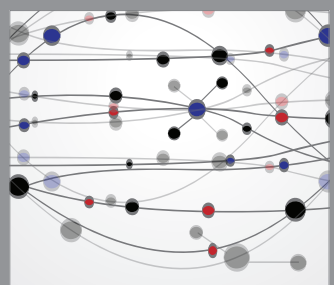

The Scientific World Journal
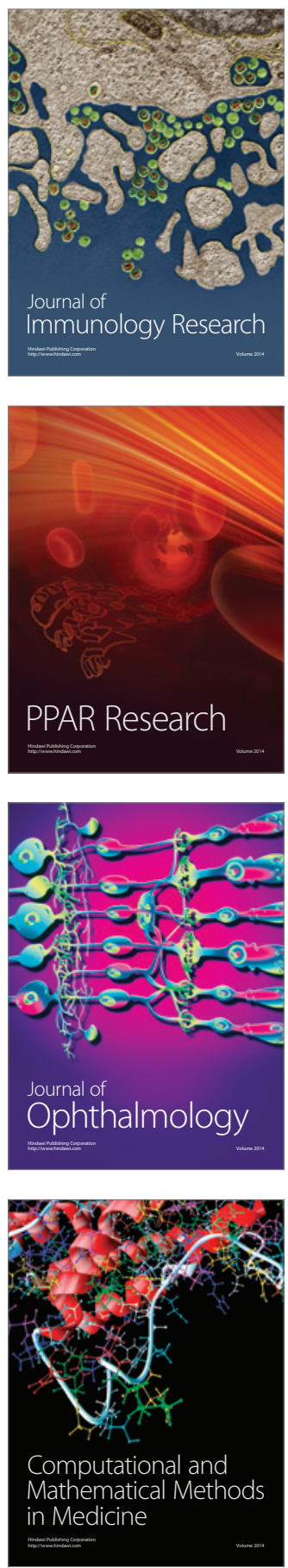

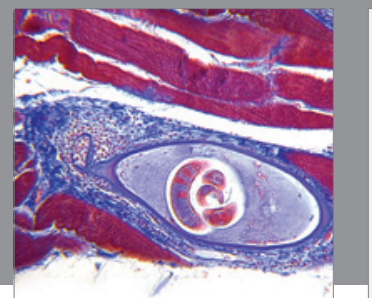

Gastroenterology

Research and Practice
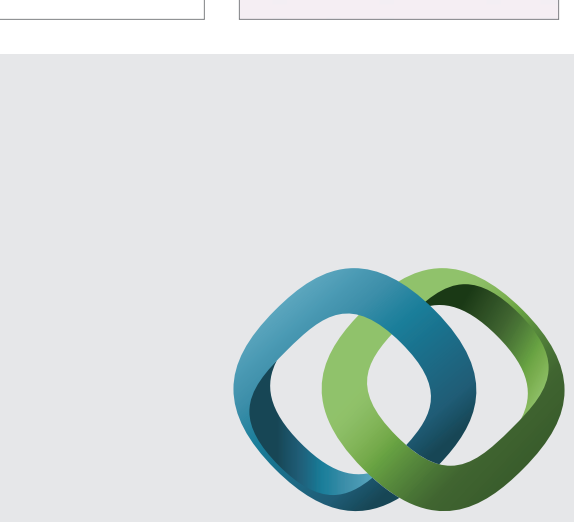

\section{Hindawi}

Submit your manuscripts at

http://www.hindawi.com
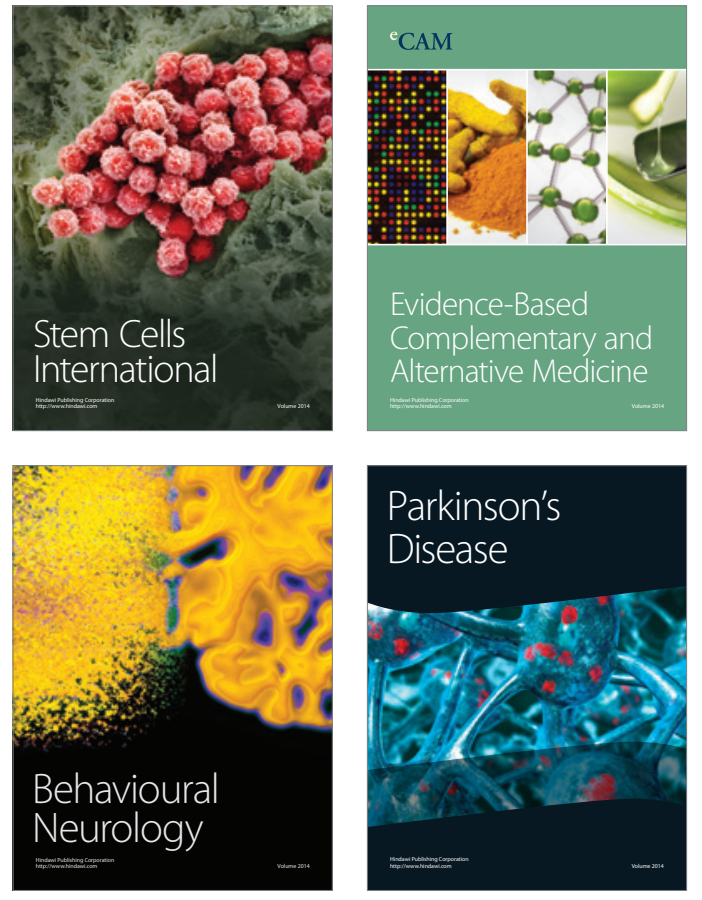
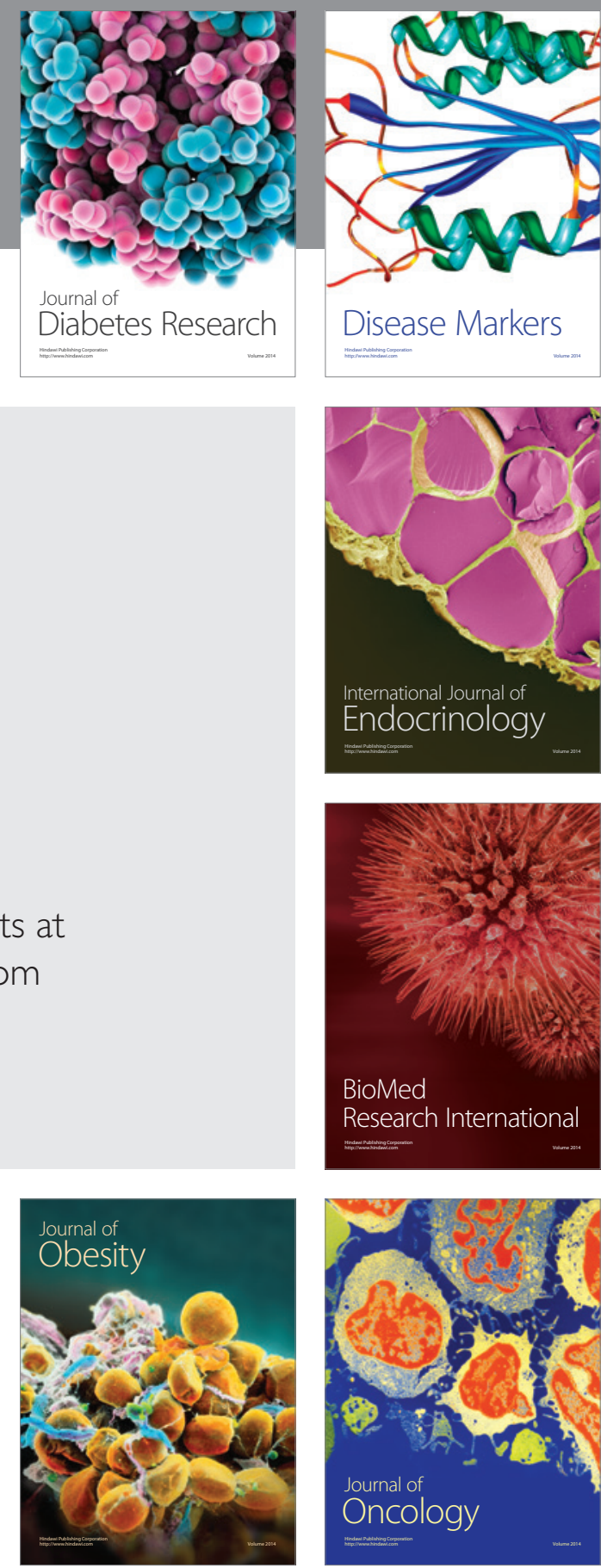

Disease Markers
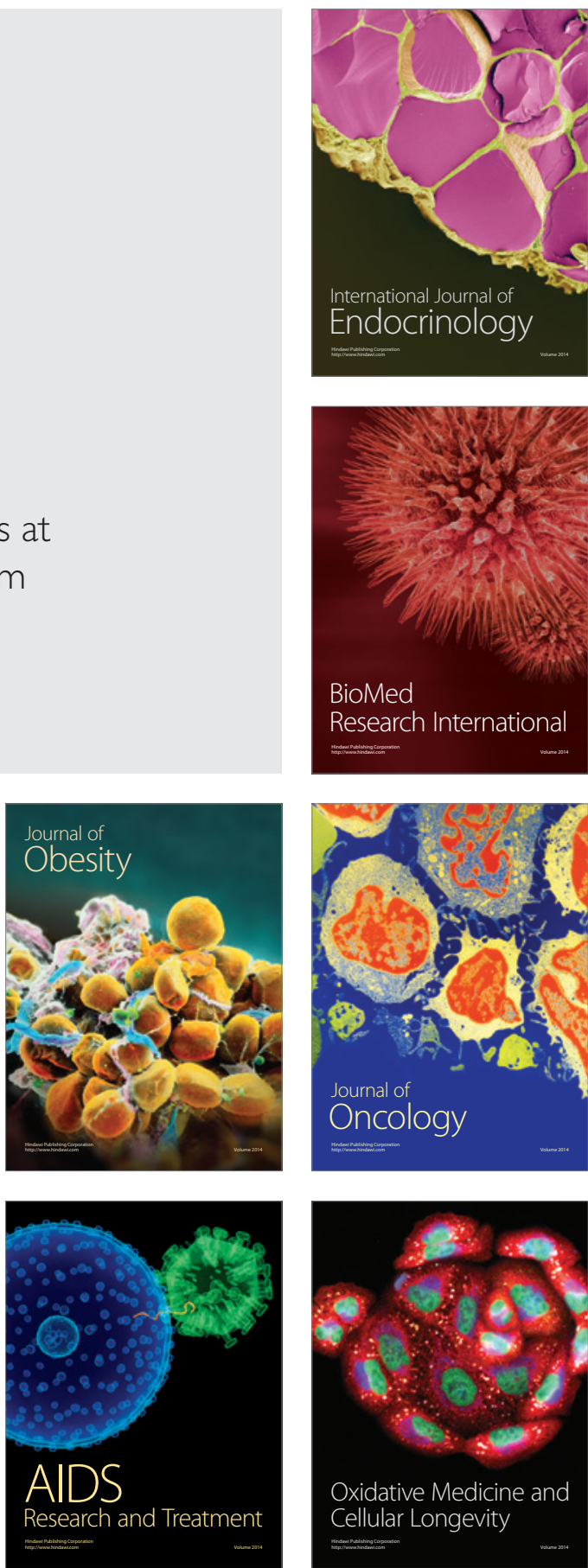